\title{
Reversible brain hypometabolism associated with central nervous system systemic lupus erythematosus
}

\author{
Jukka Kemppainen • Sami Kajander • \\ Timo Yli-Kerttula • Laura Airas • Marko Seppänen
}

Received: 1 September 2010 / Accepted: 14 October 2010/Published online: 6 November 2010

(C) Springer-Verlag 2010

A 63-year-old woman with a history of hypothyroidism and rheumatoid arthritis was referred to the internal medicine department due to progressive muscle fatigue, weight loss of $15 \mathrm{~kg}$, haemolytic anaemia, fever and memory impairment. The Mini-Mental State Examination was abnormal (MMSE 16/30). The initial brain MRI showed general cortical atrophy and FDG PET suggested a neurodegenerative disease with symmetrical severe hypometabolism in the frontal, parietal and temporal cortexes. Neurological consultation did not lead to a specific diagnosis. Later antinuclear antibody serological tests were indicative of systemic lupus erythematosus (SLE) [1-5]. The patient was treated with high-dose corticosteroids and cyclophosphamide.

After 18 months the brain scans were controlled. Anatomical and metabolic brain findings had normalized, confirming that the observed hypometabolism and atrophy were indeed related to brain manifestation of SLE and, importantly, could be reversed by efficient treatment. Cognitive performance on neuropsychological tests had also recovered. Automated

\section{J. Kemppainen $(\varangle) \cdot$ M. Seppänen}

Department of Clinical Physiology and Nuclear Medicine,

Turku PET Centre, Turku University Hospital,

Kiinamyllynkatu 4-8,

20520 Turku, Finland

e-mail: jukka.kemppainen@utu.fi

\section{S. Kajander}

Department of Radiology,

Turku PET Centre, Turku University Hospital,

Kiinamyllynkatu 4-8,

20520 Turku, Finland

\section{T. Yli-Kerttula}

Department of Internal Medicine,

Turku University Hospital,

Kiinamyllynkatu 4-8,

20520 Turku, Finland

\section{Airas}

Department of Neurology,

Turku University Hospital,

Kiinamyllynkatu 4-8,

20520 Turku, Finland comparison of the FDG PET images to a normal population with the 3D-SSP program shows the dramatic treatment effect in z-score images of surface projections.

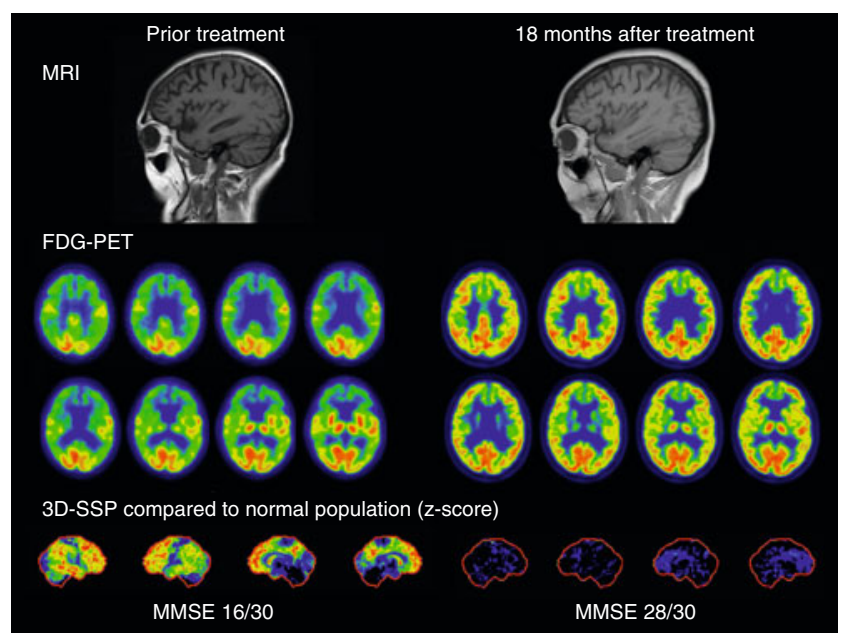

Conflicts of interest None.

\section{References}

1. Kao CH, Lan JL, ChangLai SP, Liao KK, Yen RF, Chieng PU. The role of FDG-PET, HMPAO-SPET and MRI in the detection of brain involvement in patients with systemic lupus erythematosus. Eur $\mathbf{J}$ Nucl Med 1999;26(2):129-34.

2. Minoshima S, Frey KA, Koeppe RA, Foster NL, Kuhl DE. A diagnostic approach in Alzheimer's disease using threedimensional stereotactic surface projections of fluorine-18-FDG PET. J Nucl Med 1995;36(7):1238-48.

3. Otte A, Weiner SM, Peter HH, Mueller-Brand J, Goetze M, Moser E, et al. Brain glucose utilization in systemic lupus erythematosus with neuropsychiatric symptoms: a controlled positron emission tomography study. Eur J Nucl Med 1997;24(7):787-91.

4. Sailer M, Burchert W, Ehrenheim C, Smid HG, Haas J, Wildhagen $\mathrm{K}$, et al. Positron emission tomography and magnetic resonance imaging for cerebral involvement in patients with systemic lupus erythematosus. J Neurol 1997;244(3):186-93.

5. Weiner SM, Otte A, Schumacher M, Klein R, Gutfleisch J, Brink I, et al. Diagnosis and monitoring of central nervous system involvement in systemic lupus erythematosus: value of F-18 fluorodeoxyglucose PET. Ann Rheum Dis 2000;59(5):377-85. 